\title{
A Roundtable of Innovative Leaders in Medical Education
}

\author{
By André-Jacques Neusy, MD, DTM\&H and Bjorg Palsdottir, MPA
}

In 2008, the Global Health Education Consortium (GHEC) created the Training for Health Equity Network (THEnet), bringing together schools in different parts of the world that share a core mission: to recruit students from, and produce physicians for, underserved communities. In determining the competencies such physicians will need, these schools also share an approach to medical education that looks beyond the traditional curriculum and seeks the involvement of communities and other stakeholders. Their input helps define the knowledge, skills, and attitudes around which new curriculum is built, and helps guide the selection of educational methodologies, taking into account context and resource constraints.

The nascent THEnet now has a nucleus of eight member schools, each dedicated to fulfilling a strong social accountability mandate. The network is designed to assist them by providing a collaborative platform conducive to experimentation; dialogue; and creation and sharing of tools, experiences and evidence. It will also support systematic outcome evaluations, innovation, and joint research to strengthen the knowledge base on successful strategies for increasing the number

\section{MEDICC Review: What do you see as the key competencies doctors must acquire to meet health needs in the twenty-first century, and how are your schools trying to develop these?}

Richard Murray (JCU): The James Cook University curriculum has a strong emphasis on general medicine, population health, and team care. The rise of chronic disease, ageing populations and cost constraints in health care systems requires doctors who have a population perspective. In practice, this means a greater focus on clinical governance, development of care protocols, appraisal of evidence in the local context, quality enhancement activities, and use of information and communication technology.

Medical graduates of the twenty-first century will increasingly assume the role of medical experts in a team setting. For individual patients, doctors will be principally responsible for the procedurally or cognitively more complex aspects of care rather than the routine aspects. This implies expanded and flexible clinical roles for other members of the health care team and greater responsibility for patients in managing their own care. We need multiprofessional care, not the 'multiple professional' care we see in the trend driven by excessive specialization. A resurgence of the generalist approach is required across all medical disciplines, with the boundaries of many disciplines being redrawn.

Fortunato Cristobal (AZU): Competencies need to be developed that address the broader issues of population health. The traditional model is too biologically-oriented and is limited by its focus on individual health. This approach has become too expensive, leading to late intervention in the health care spectrum and, at worst, failure to solve health problems. Therefore, the social and biological models of health care must be given equal emphasis. and quality of doctors in neglected communities. By bringing these schools together, developing synergy among them and publishing their results, THEnet hopes to more broadly promote the transformation of medical education and medical practice into more socially accountable endeavors that improve health system equity and performance.

Leaders from six of these innovative medical schools spoke with the Guest Editors of this issue of MEDICC Review, a conversation we bring you below. They are: Dr Juan Carrizo, Rector of the Latin American Medical School (ELAM), Cuba; Dr Fortunato L. Cristobal, founding Dean of the School of Medicine at Ateneo de Zamboanga University (AZU), the Philippines; Dr Pedro Díaz, member of the National Academic Coordinating Committee of the National Training Program for Comprehensive Community Physicians (NTPCCP), Venezuela; Dr Richard Murray, Dean and Head of the School of Medicine and Dentistry, James Cook University (JCU), Australia; Dr Roger Strasser, founding Dean of Northern Ontario School of Medicine (NOSM), Canada; and Dr Paul Worley, Dean of Flinders University School of Medicine (FUSM), Australia

Pedro Díaz (NTPCCP): Doctors need five fundamental competencies to address a society's health needs: they must be able to provide high-quality care; promote individual and community health; develop effective preventive approaches; rehabilitate the patient to good health; and act as an agent of social transformation. Our integrated approach is based on primary health care principles, focusing on the needs of the individual, his/her family and community within their sociocultural and economic context. Doctors need to be able to conduct a community diagnosis; design interventions; and understand the social, economic and spiritual impact of an intervention on all sectors of the community.

Roger Strasser (NOSM): Given the complexity of life in the twenty-first century, people need doctors who are able to listen and respond to their health needs. The Northern Ontario School of Medicine aims to graduate doctors who have the skills and capabilities to pursue medical careers anywhere in Canada or the world, but who have a special affinity for, and comfort with, providing health care in the geographically, socially and culturally diverse communities of Northern Ontario.

Juan Carrizo (ELAM): The real question is, what kind of doctor is needed? A well-trained doctor, of course, but also a doctor who is grounded in the community, who treats the individuals and families that make up the community, and who is intimately familiar with the health profile of the local population. The backbone of our program is its emphasis on quality primary care coverage for 100 percent of the population. Our approach is based on an integrated program with many hours dedicated to epidemiology, hygiene and similar subjects, as well as psychology, social medicine, management and other social sciences. We think that students who receive this type of training are better prepared for their 
future as doctors; they have a better understanding of health and well-being, and are more motivated to help those around them.

Paul Worley (FUSM): I prefer to see the core competencies, on one hand, as the 'head' knowledge and skills required by all medical schools and, on the other hand, the 'heart' knowledge demonstrated by an attitude of service and a willingness to practice where and when the community needs you, not just where and when it is convenient. This 'heart' knowledge is what our students learn through experience in our rural and remote programs.

\section{MEDICC Review: What are three important approaches that distinguish your medical school from other more traditional Flexnerian programs in your region?}

Fortunato Cristobal (AZU): Rather than teaching subject courses separately, our curriculum integrates biological and social health care models, and also balances individual and population health. We use problem-based and community-oriented learning along with competency-based evaluations. From day one, students are exposed to patients and the community. Each student is placed in a community where they spend one month at the end of every semester until graduation, in addition to the entire 4th year community clerkship. During this community exposure time, students make health interventions, implement community health programs, and conduct interventional research using an intersectoral approach. Half of the third year is spent in the hospital for continuous clinical exposure. Half of the fifth year is again spent in a hospital setting, while the other half is spent rotating in various clinical settings, such as a community emergency health clinic, provincial hospital, or community health station. This rotation exposes students to the realities of both the health system and health practice in the region.

Pedro Díaz (NTPCCP): The first difference is that our medical school is not set apart from the community. We have no campuses per se. The typical learning environment is one of the poor communities from which the students are recruited. This is quite different from the campus- and hospital-centered Flexnerian model. A century ago, Abraham Flexner made a very important contribution to modern medical education by insisting on a scientific foundation. But this model needs revisiting to take into account the socioeconomic, cultural and scientific changes that have occurred since that time.

The second major difference is the type of faculty and their relationship to the curriculum and to the students. The Flexnerian model calls for teachers with specific competencies in their respective specialties - physiology, cardiology or biochemistry, for example - each of whom teaches his or her subject matter in a separate, vertical manner. However, this model doesn't reflect the evolving health needs of populations. Epidemiological patterns are changing as society changes due to the pressure of globalization and continuous alterations in the economic and sociopolitical landscape. Our curriculum is an evolving process that integrates the basic socio-medical, clinical-epidemiological sciences with public health, horizontally and vertically, throughout the six-year program. In our model, all faculty members are both medical practitioners and trained medical educators. Each professor teaches all subjects in an integrated fashion, using real cases from the community where he or she practices. Throughout the curriculum, the physician-teacher reinterprets basic sciences from clinical and public health perspectives. In a sense, the professor goes back to medical school with his/her students.

NTPCCP students acquire clinical and public health competencies early, because they are exposed to real patients from the beginning of the program. The student is both observer and active co-participant in the care of a patient, his/her family and community: taking histories, progressively acquiring clinical skills, and doing community health surveys and other research leading to co-intervention with the community.

Paul Worley (FUSM): First, nearly half of our students undertake their major clinical clerkships in indigenous, rural or remote settings over a geographic region spanning 3,500 kilometers. Second, primary care is increasingly seen as the principal educational setting, with specialists providing 'consultant' educational input, similar to the 'consultant' clinical input they provide to patient care. Lastly, higher percentages of students of rural/remote origin are attracted to our course. I see our approach as the logical extension of the Flexnerian model for the twenty-first century. As more medical care shifts to ambulatory and community settings, and as the science underpinning health is broadened to include social and population perspectives, the logical Flexnerian response for undergraduate-level medical education is to move increasingly away from tertiary centers.

Juan Carrizo (ELAM): We want our students to understand that medicine is not just curative; that it is a matter of treating the person, not the disease. Two main factors particular to ELAM are helping us achieve this. First, as soon as students enter the program, they begin studying the health problems of a community at a "university polyclinic". Second, we have replaced the teaching of sciences in isolation - anatomy one semester, microbiology the next - with a morphophysiological pedagogical approach, which enables students to better analyze, problem-solve and integrate knowledge in a cumulative, comprehensive way. We design our courses so that everything is connected, making it easier to understand the patient as a whole, while being careful not to compromise the quality of the students' scientific training. We have found that students absorb scientific knowledge better with this methodology and are better prepared to solve clinical health problems, pursue research and develop professionally.

Roger Strasser (NOSM): NOSM recruits students from Northern Ontario or similar Northern, rural, remote, Aboriginal, Francophone backgrounds. The school provides a learner-centered, clinically-driven undergraduate medical education program featuring Distributed Community-Engaged Learning, an instructional model that allows widely distributed human and instructional resources to be used independent of time and place in community partner locations across a large geographic region. Instead of traditional subjects, the four-year undergraduate program is organized around five themes: Northern and Rural Health; Personal and Professional Aspects of Medical Practice; Social and Population Health; Foundations of Medicine; and Clinical Skills in Healthcare.

Richard Murray (JCU): First, our training model incorporates strong themes related to rural and remote health, Indigenous Australian health, tropical medicine and, more broadly, the needs of underserved populations located in the Northern Territories. Second, our curriculum is comprehensive with a strong biomedical 
and social science base. It provides substantial clinical experience across the six-year program, including at least 20 weeks spent in small rural towns or remote communities. We believe that confident, work-ready graduates are more comfortable choosing low-resource settings and isolated practice. Lastly, we are readdressing inequity in educational opportunities by targeting recruitment predominantly towards secondary school graduates, selected on the basis of rurally-weighted academic performance combined with an interview involving local community, health professionals, and academics. There is also a special selection process for Indigenous Australian students. This has given us a unique student demographic in Australia in terms of rural origin and socio-economic background.

MEDICC Review: What role should a medical school play in health system development, and what impact do you see your medical school having on health in your community?

Roger Strasser (NOSM): I see medical schools playing a critical role in health system development by graduating suitably trained doctors and through research. NOSM has a formal collaboration with regional health authorities in Northern Ontario, known as Local Health Integration Networks, to improve health care and enhance the health workforce in Northern Ontario.

Pedro Díaz (NTPCCP): Our medical education program is closely associated with the development of a new public health system in Venezuela. The NTPCCP originated with the Barrio Adentro project, initiated in 2003 by the Venezuelan government to establish a new primary care-oriented health system, replacing a system that excluded more than 70 percent of the population. Barrio Adentro began by setting up a network of consultorios, or outpatient clinics, in urban and rural communities throughout the country, which now serve as hubs of our medical education efforts. Our students and professors are providing quality care all over the country and are also involved in a broad range of activities aimed at strengthening the system, such as conducting population health surveys to identify health risks, and intervention opportunities for improving community health status. A nationwide network of diagnostic and rehabilitation centers was also created, giving our physicianteachers a platform for providing an integrated approach to general medicine. The students we train in comprehensive general medicine (family medicine) will be the generation that staffs both the free primary health care services and the more specialist-oriented secondary health care facilities.

Paul Worley (FUSM): Medical schools and health services need to develop symbiotically. This has been the case for Flinders in both our tertiary and rural settings. In the 1970s, construction of our school with its associated teaching hospital provided a significant boost to the underserved communities in the south of Adelaide. Our rural programs provided a significant retention incentive for experienced rural clinicians, as they were able to diversify into an academic role in their own communities. Our expansion into the Northern Territory has transformed the Royal Darwin Hospital from a very difficult place to recruit high quality doctors to a place offering prestigious academic posts that attract competition from doctors wishing to work there.

Juan Carrizo (ELAM): Many of ELAM's graduates from Latin America are already working in their communities, providing care for people who never received care before - and in the local lan- guage! These specialists in family medicine learn not only obstetrics, cardiology, anesthesiology, pediatrics and the like; they also learn to use appropriate technology. This is a tremendous asset for their countries, because when they return home, they infuse the health system with capable, highly-trained doctors - at no cost to the government.

Fortunato Cristobal (AZU): We produce graduates with competencies that are appropriate for our local health needs and health care system. Eventually these doctors will occupy key positions in the health system and, because they are problem solvers, they will eventually promote change in the system. In the past, biological and individual approaches to solving health problems upheld the system, even when the causes of ill health were social. Because of our graduates' balanced social orientation to health care, we are already beginning to see the paradigm shift.

Richard Murray (JCU): We are a medical school that arose from community struggle and that values social justice, innovation and excellence. Already the program has aided the transformation of provincial hospitals from service providers to teaching and research-rich environments that more easily attract and retain clinicians and academics. We have doubled the number of junior doctors in a labor-starved system and have seen many struggling rural health facilities transition to vibrant 'teaching health services'. Students, and now our graduates, have been important in renewing optimism about the future of health care among rural doctors and local communities.

\section{MEDICC Review: What are the two greatest challenges facing your school?}

Roger Strasser (NOSM): The greatest challenges for NOSM involve sustainment. Continuation of the school's success as an innovator in medical education is one such challenge. Another will be to maintain and further develop the active participation of community members and clinicians across all of the school's 70 teaching-learning sites.

Fortunato Cristobal (AZU): Recognition has been our greatest challenge. While we have achieved recognition internationally, we have yet to receive recognition or much support from the government. The courage to shift away from the traditional approach has always been met with scorn, ridicule and outright opposition from the medical profession itself, as well as from government institutions, which have been reluctant to give us the mandate to pursue and probe innovative approaches.

Richard Murray (JCU): We need a regional pipeline for specialist training. Vocational training across medical specialties in Australia is built on urban models with centralized governance structures. We are working on tripling regionally-based medical specialist training in tropical Australia. This will require substantial new investment in training and registrar (resident) salaries; creativity to obtain accreditation of training posts; and a greater regional voice in trainee selection. Without this, graduates will face pressures to relocate to cities. We also need to develop a research infrastructure for northern Australia. Capitalizing on the unique opportunities afforded by our geographical location, distinct population and health challenges requires a lump sum for research spending, not piecemeal funding. 
Juan Carrizo (ELAM): Our biggest challenge is heterogeneity, which is also ELAM's strength and one element that distinguishes us from other schools. We are always working to improve the student selection and intake processes. For example, using the same selection process for each country is not practical, because each has its own educational structure and requirements. Add to this different customs and culture, language and habits, and it gets really complicated. To improve the intake process, we developed a pre-med course designed to help students adjust to the new setting and prepare them to begin their medical coursework at more or less the same level. The pre-med course can last one or two semesters, depending on how much help the student needs.

Our other major challenge is achieving recognition of the ELAM degree in our graduates' countries of origin. We have succeeded in some countries; in others, recognition has been granted on an individual basis; and in others, the recognition procedure is in process.

Paul Worley (FUSM): Our first main challenge is translating our rural clinical school model into a model for underserved urban areas. The second is finding academic leaders with outstanding teaching and research track records to guide our process. Given the extraordinary difference between clinical and academic salaries, choosing to teach is almost a philanthropic career move.
Pedro Díaz (NTPCCP): Our ongoing challenge is achieving excellence in providing quality comprehensive care - and not just medical care - to the Venezuelan people. Our broad approach to medical education strives to make an impact on the quality of life of each individual and community by identifying and eliminating health risks, and by creating conditions conducive to a healthy lifestyle.

The second challenge is consolidating and maintaining the goal of primary health care as the foundation of the health system. Following the Alma-Ata Declaration, which called for "health for all," many governments and "experts" developed an oppositional strategy that cast doubts on the costs and feasibility of this important goal. This resulted in a distortion of Alma-Ata and the continuation of the vertical disease-oriented approach.

\section{MEDICC Review: What defines success for your school?}

Fortunato Cristobal (AZU): Success is achieving our mission and vision. We do not compete to achieve international standards based on traditional educational approaches. Instead, we measure ourselves against our own key indicators for success, which include producing graduates with a balanced perspective toward biological and social health care models; effecting change through policymaking by doctors holding key positions in the

\begin{tabular}{|c|c|c|}
\hline School & Location & $\begin{array}{c}\text { Year } \\
\text { Founded }\end{array}$ \\
\hline $\begin{array}{l}\text { Flinders University School } \\
\text { of Medicine } \\
\text { http://som.flinders.edu.au/ }\end{array}$ & $\begin{array}{l}\text { University campus in Adelaide, } \\
\text { Australia; rural and remote } \\
\text { programs based in the } \\
\text { Northern Territory, Victoria, } \\
\text { and South Australia }\end{array}$ & 1975 \\
\hline
\end{tabular}

\section{Program}

Four-year graduate MD program. Parallel Rural Community Curriculum (PRCC) introduced in 1997; Northern Territory Clinical School (NTCS) created in 1998

\begin{tabular}{|c|c|c|c|c|}
\hline & & & & \\
\hline $\begin{array}{l}\text { Ateneo de Zamboanga } \\
\text { University School of Medicine } \\
\text { http://som.adzu.edu.ph }\end{array}$ & $\begin{array}{l}\text { Zamboanga City, Mindanao, } \\
\text { the Philippines }\end{array}$ & 1993 & $\begin{array}{l}\text { Four-year graduate MD training, } \\
\text { about } 50 \% \text { community-based. } \\
\text { Fifth-year Masters in Public Health } \\
\text { (MPH); Masters in Health Professions } \\
\text { Education also offered }\end{array}$ & $\begin{array}{l}\text { Current total enrollment: } \\
\text { approximately } 125\end{array}$ \\
\hline $\begin{array}{l}\text { Latin American Medical School } \\
\text { (Escuela Latinoamericana } \\
\text { de Medicina - ELAM) } \\
\text { http://www.elacm.sld.cu }\end{array}$ & Havana, Cuba & 1999 & $\begin{array}{l}\text { Six-year undergraduaute MD program; } \\
\text { two years of basic science, followed } \\
\text { by four years of clinical rotations; } \\
\text { some students spend year } 6 \text { in } \\
\text { supervised practice in their home } \\
\text { country }\end{array}$ & $\begin{array}{l}\text { Current total enrollment: } \\
\text { approximately } 10,000 \\
\text { Total graduates } 2005-2008 \text { : } \\
5,945\end{array}$ \\
\hline $\begin{array}{l}\text { James Cook University School } \\
\text { of Medicine } \\
\text { http://www.jcu.edu.au/medicine }\end{array}$ & Townsville, Northern Australia & 2000 & $\begin{array}{l}\text { Six-year undergraduate MD program; } \\
\text { years } 1-3 \text { university based; years } 4-6 \\
\text { in health care facilities, including } 20 \\
\text { weeks of clinical training in small rural } \\
\text { and isolated settings }\end{array}$ & Current total enrollment: 609 \\
\hline $\begin{array}{l}\text { Northern Ontario School } \\
\text { of Medicine } \\
\text { http://www.normed.ca/ }\end{array}$ & $\begin{array}{l}\text { Thunder Bay and Sudbury, } \\
\text { Ontario, Canada }\end{array}$ & 2004 & $\begin{array}{l}\text { Four-year graduate MD program } \\
\text { with four- and eight-week sessions in } \\
\text { rural communities in years } 1 \text { and } 2 \text {, } \\
\text { respectively; year } 3 \text { entirely based in } \\
\text { a rural family practice setting }\end{array}$ & $\begin{array}{l}\text { Current enrollment: } 56 \text { in each } \\
\text { year; first MD graduates in } \\
2009 \text {, as well as first } 30 \text { family } \\
\text { medicine residents }\end{array}$ \\
\hline $\begin{array}{l}\text { Barrio Adentro University } \\
\text { Comprehensive Community } \\
\text { Physician Training Program }\end{array}$ & $\begin{array}{l}\text { Venezuela, "open campus" in } \\
\text { association with six national } \\
\text { universities }\end{array}$ & 2004 & $\begin{array}{l}\text { Six-year undergraduate } \\
\text { Comprehensive General Medicine } \\
\text { training, entirely community-based in } \\
5,131 \text { accredited community clinics } \\
\text { and other health care facilities, } \\
\text { and } 855 \text { multipurpose classrooms } \\
\text { distributed throughout all } 24 \text { states }\end{array}$ & $\begin{array}{l}\text { Current total enrollment: } \\
\text { approximately } 22,000 ; \\
\text { first graduating class in } 2010\end{array}$ \\
\hline
\end{tabular}


health system; delivering health care that is sensitive to the cultural, economic, social, and political realities of the region, and that is capable of solving regional health problems. Just as all sectors and stakeholders in the health system should be involved in our educational program, the academic program should also be deeply involved in every aspect of health care, from training and research to delivery and system development.

Juan Carrizo (ELAM): Since 2005, we have graduated nearly 6,000 well-trained doctors. That is success. The impact our graduates are having on health in their home countries is also an important indicator. It is so satisfying to see these young men and women helping their people and to see their home communities benefit from ELAM's work. They are terrific role models, and now other young people from their communities want to study at ELAM.

Another indicator of success is our graduates' feeling of connection to ELAM. They have taken ownership of this project. They feel connected to each other as well, no matter what country they are from; the sense of solidarity and internationalism they share is impressive. This is a great motivator for us - the administrators and faculty; it helps us know that what we're doing is making an impact.

Roger Strasser (NOSM): In the long term, NOSM's success will be measurable improvements in the health of the people and communities of Northern Ontario. Medium-term indicators of success include NOSM graduates choosing to practice in Northern Ontario along with substantial research output, which contributes to improved health care and health outcomes.

Paul Worley (FUSM): Success for us means that health services and clinicians in all our regions will ask us to send them more students. This would indicate that they have enough clinical capacity and that they see our students as integral to the long-term sustainability of the clinical service they provide. It also means that the communities we serve acknowledge that our research is making a difference in the clinical care they receive.

Richard Murray (JCU): We are successful when the majority of our graduates choose a career in northern or regional Australia. While their vocational training will span the full range of medical disciplines, many will have chosen rural generalist practice and regional generalist specialties. Graduates will be distinguished both by their clinical competence and an informed commitment to social justice and will assume leadership roles based on both. The expanded research endeavor will focus on applied research with underserved populations as well as unique opportunities for biomedicine in the tropics. Other achievements will include innovation in the health workforce and health services, including strengthened team roles and opportunities for a broader base of participation in health care.

Pedro Díaz (NTPCCP): Success comes down to how many lives have been saved. This indicator is controversial outside disaster settings, where it is most commonly used. Nevertheless, we endorse this indicator of success, given that, prior to the Barrio Adentro mission, the majority of the Venezuelan population had no access to care. Our medical education program, our students and faculty, are integral parts of the development of the public health system in Venezuela, so success will be achieved when these activities benefit all Venezuelans.

\section{MEDICC Review: What single piece of advice would you give others who want to develop medical education programs to train doctors to provide care in underserved communities?}

Paul Worley (FUSM): Start small, 'below the radar', and with enthusiastic volunteer students and faculty. Use reliable statistical measures to compare outcomes with the existing 'gold standard'.

Roger Strasser (NOSM): I recommend developing medical education programs that are grounded in underserved communities. This involves the active participation of community members in curriculum development and delivery, so that students undertake their clinical learning in the context of these same communities.

Juan Carrizo (ELAM): We know that changing a political or social system is harder than changing a curriculum. My advice, then, is not to give up if the government is not supportive; university authorities have to apply their own political will within their institutions. I believe that a medical school that adheres to the tenets of Alma-Ata and the Millennium Development Goals can be developed in any country, regardless of the political or social system. Those of us working towards the goals of Alma-Ata need to work better together. We have to reach out to foundations, organizations, governments and individuals - not only when there is political will, but also when there is none.

We do not want an ELAM monopoly - we want many ELAMs. The demand exists; we receive 20 times the number of applications as there are slots available for admission. If universities could graduate $25 \%$ more doctors trained in this philosophy by 2025 , together we could fulfill the world's need for primary health care practitioners. For health authorities, academics and university administrators, the key is focusing on community health.

Richard Murray (JCU): Student selection is vital, especially engaging high-school leavers from underserved groups. This, combined with a suitable curriculum and clinical immersion with inspiring preceptors and teachers, establishes a school culture that attracts students and faculty who share common values. Our regional base and partnering with community organizations are also critical factors.

Pedro Díaz (NTPCCP): You have to learn to unlearn! There is a growing recognition among medical educators and practitioners that the traditional Flexnerian model still used by most medical schools is not preparing medical practitioners to solve the needs of the people they serve. This recognition is what led Cuban educators to unlearn and then rebuild from the Flexnerian model to develop a more holistic approach to medical education that integrates basic medical and social sciences with public health and humanities. This new value-driven model is reinforced by pedagogical methodologies that allow medical students to become self-directed learners and discoverers of knowledge in an ongoing process.

Fortunato Cristobal (AZU): Start with a relevant, well-defined educational philosophy drawn from people's empowered participation. Do not compromise and do not be intimidated! 


\section{Erratum}

The correct website for the Ateneo de Zamboanga University School of Medicine in the Philippines is: http://som.adzu.edu.ph 